\title{
Saccadic Eye Movements Illuminate Chronometry of Perceptual Localization
}

\author{
Florian Ostendorf \\ Klinik für Neurologie, Charité - Universitätsmedizin Berlin, D-10117 Berlin, Germany \\ Review of de'Sperati and Baud-Bovy (http://www.jneurosci.org/cgi/content/full/28/17/4317)
}

Mental chronometry originated in the late 1800 s as an experimental approach that used measured response times in perceptual-motor tasks as a tool to infer underlying cognitive operations from overt behavior (Donders, 1969). In a recent article published in The Journal of Neuroscience, de'Sperati and Baud-Bovy (2008) exploit a chronometric approach to tackle a longstanding issue in perceptual neuroscience: to what degree is vision for directed motor action and vision for perception processed in separate neural pathways? In an influential and widely disputed study in 1992, Goodale and Milner proposed such a neuroanatomical specialization, mainly based on findings in neuropsychological patients (for a recent update, see Milner and Goodale, 2008). A growing body of studies in neurologically healthy subjects followed up on this proposal by asking whether visual illusions can affect directed motor actions, such as grasping movements. Up to now, experimental findings have remained equivocal, and different theories have been proposed to account for the discrepant results.

Maybe the most challenging perceptual-motor system for the concept

Received June 30, 2008; revised July 22, 2008; accepted July 22, 2008.

This work was supported by German Federal Ministry of Education and Research Grant 01 GW0653 (Visuospatial-Cognition).

Correspondence should be addressed to Florian Ostendorf, Klinik für Neurologie, Charité - Universitätsmedizin Berlin, Charitéplatz 1, D-10117 Berlin, Germany. E-mail: florian.ostendorf@charite.de.

DOI:10.1523/JNEUROSCI.3005-08.2008

Copyright $\odot 2008$ Society for Neuroscience $\quad 0270-6474 / 08 / 289090-02 \$ 15.00 / 0$ of functional perception-action dissociations is provided by the oculomotor system. Here, the peripheral sensor itself, the retina, is constantly displaced by the oculomotor action, leading to a most intimate interplay between visual perception and oculomotor action. de'Sperati and BaudBovy (2008) decided to use exactly this system to probe the susceptibility of perception and action to one specific visual illusion, the illusory displacement of a flashed stimulus because of nearby motion signals (Whitney and Cavanagh, 2000; Durant and Johnston, 2004). In their study, de'Sperati and Baud-Bovy (2008) asked six normal subjects to fixate on a central cross. A circular arc was then presented at $5^{\circ}$ eccentricity either right or left of central fixation. After a variable period, this arc started revolving at constant speed $\left(200^{\circ} / \mathrm{s}\right)$ in either the clockwise or counterclockwise direction. Just at motion onset, a small white spot was flashed slightly more eccentric besides the arc, at the trailing edge, center part, or leading edge [de'Sperati and Baud-Bovy (2008), their Fig. 1 (http://www.jneurosci.org/ cgi/content/full/28/17/4317/F1)]. In the main condition used for probing perceptual localization, subjects were instructed to report the apparent flash position by rotating a pointer while maintaining central fixation. Similar to published findings in the literature (Whitney and Cavanagh, 2000; Durant and Johnston, 2004), the apparent flash direction systematically shifted $\sim 10^{\circ}$ in the direction of the nearby motion signal.
To probe oculomotor localization in this task, subjects were instructed to saccade from the central fixation to the flashed dot position. Here, saccade endpoints also exhibited a systematic bias in the direction of motion, demonstrating that visual processing for oculomotor actions was also affected by the motioninduced mislocalization. However, the average direction error amounted to only $4.13^{\circ}$, indicating a weaker affect on oculomotor responses than on perceptual localization. Whereas such a differential susceptibility of perceptual versus motor performance to visual illusions has been demonstrated in other modalities, the specific task allowed de'Sperati and BaudBovy to go one decisive step farther in their analysis. Plotting the directional errors against the corresponding saccadic reaction times (SRTs) revealed a clear dependency of the illusory bias on the viewing time preceding saccade execution: for saccades with short SRTs of $\sim 200 \mathrm{~ms}$, virtually no illusory shift in saccade endpoints was observed (mean directional error, $0.28^{\circ}$ ). Saccades with longer SRTs showed a gradual build-up of directional endpoint errors and saccades with SRTs of $400 \mathrm{~ms}$ or more exhibited an almost identical strength of the visual illusion compared with the perceptual condition [de'Sperati and Baud-Bovy (2008), their Fig. 2 (http://www.jneurosci.org/cgi/ content/full/28/17/4317/F2)].

In a second oculomotor condition, task instructions prioritized urgency, and the stimulus sequence was changed with a 
brief temporal gap introduced between an offset of central fixation and flash presentation. With these experimental manipulations, the authors induced faster saccadic responses and even so-called "express" saccades with SRTs shorter than $120 \mathrm{~ms}$ after flash presentation. The authors first confirmed that directional errors for the two saccade conditions were not significantly different for trials with overlapping SRTs. Pooling across the two saccade conditions then revealed the full pattern of saccadic directional changes over the first half second after flash presentation for the three different flash positions [de'Sperati and Baud-Bovy (2008), their Fig. 3 (http://www.jneurosci.org/ cgi/content/full/28/17/4317/F3)]: average direction errors indicated that express saccades with very short SRTs were anticipatory, because they did not account for different flash positions. Starting with SRTs of more than $\sim 100 \mathrm{~ms}$, saccade directions gradually reflected the corresponding flash positions. For saccades with SRTs longer than $\sim 250 \mathrm{~ms}$, saccade directions then gradually became affected by the illusory shift in motion direction. This motion-induced mislocalization plateaued with SRTs of $\sim 450 \mathrm{~ms}$ or more. de'Sperati and Baud-Bovy (2008) fitted the time course of saccadic directional errors with the sum of two logistic functions, which seems to provide a plausible model for the evolving dynamics of directional errors at least for flashes presented at the center and the leading edge of the nearby arc.

de'Sperati and Baud-Bovy (2008) take their findings as evidence for a transient dissociation of perception and action, because saccades with SRTs of $\sim 200 \mathrm{~ms}$ escaped the illusory mislocalization while being clearly target directed. Their interesting finding might, however, emphasize the remarkable flexibility of the oculomotor system rather than support the view of functionally separate pathways for visual perception and oculomotor action. Previous studies have shown that an illusory mislocalization in perceptual tasks is determined by motion signals occurring between $60 \mathrm{~ms}$ (Durant and Johnston, 2004) and $200 \mathrm{~ms}$ (Whitney and Cavanagh, 2000) after the flash presentation. Sub- tracting a saccadic "dead-time" of $\sim 80 \mathrm{~ms}$ (i.e., the time period in which incoming visual information cannot anymore modify an oculomotor command transmitted downstream) from the latency of $\sim 250$ ms (the SRT value in which the illusion started to affect saccade endpoints in the current study) might suggest a similar time course of the motion-induced mislocalization in both the perceptual and oculomotor domain. Although such a calculation across tasks and studies can only be illustrative, it would definitively be interesting to recover the temporal dynamics of the motion-induced mislocalization in the perceptual condition of the study by de'Sperati and Baud-Bovy (2008). Restricting viewing times in the perceptual condition may yield a similar build-up of the illusion as elegantly sampled by variable reaction times in the oculomotor condition. Such a result would then question the idea of an "asynchrony between seeing and looking" and, as also suggested by the authors, support the notion that saccadic eye movements with variable SRTs sampled the entire build-up of the visual illusion, whereas in the perceptual condition mainly the final plateau state of the illusion was captured.

Under this perspective, the study of de'Sperati and Baud-Bovy (2008) highlights a peculiar characteristic of the oculomotor system. Recent studies agree that subjects may deliberately limit the time of information accumulation for oculomotor decisions (Caspi et al., 2004; Ludwig et al., 2005; Kiani et al., 2008): visual integration can be terminated well before the saccadic dead time (Caspi et al., 2004; Ludwig et al., 2005) and such a preliminary termination of visual integration was even observed when observers were required to view the entire stimulus and postpone their oculomotor action (Kiani et al., 2008). These findings suggest that the underlying decision process terminates when accumulated sensory evidence reaches a critical level, although longer accumulation times may have further improved response accuracy. Oculomotor actions may exhibit such a speed bias, because rapid gaze orienting toward relevant aspects of a visual scene may be crucial in real life. In this context, it is interesting to note that the cost of inaccurate primary saccades may be reduced by rapid corrective saccades that take into account visual information that is sampled within the dead time of the primary saccade (Caspi et al., 2004).

Here, the study of de'Sperati and Baud-Bovy (2008) further emphasizes the remarkable flexibility of the oculomotor system: on the one hand, saccadic eye movements with short SRTs can be based on a first, feedforward swipe of visual information. Paradoxically, such a limited visual integration time led to more accurate performance in the present study, leaving saccade endpoints unaffected by the motion-induced illusion. However, saccades can also take into account the result of a more advanced visual integration process, presumably mediated by reentrant processing loops from higher visual areas. These findings limit the generalization of "single-shot" accounts of visual integration for saccadic eye movements (Ludwig et al., 2005) but will likely stimulate a further behavioral exploration of the subsecond dynamics of perceptualmotor decision making.

\section{References}

Caspi A, Beutter BR, Eckstein MP (2004) The time course of visual information accrual guiding eye movement decisions. Proc Natl Acad Sci U S A 101:13086-13090.

de'Sperati C, Baud-Bovy G (2008) Blind saccades: an asynchrony between seeing and looking. J Neurosci 28:4317-4321.

Donders FC (1969) On the speed of mental processes. Acta Psychol 30:412-431.

Durant S, Johnston A (2004) Temporal dependence of local motion induced shifts in perceived position. Vision Res 44:357-366.

Kiani R, Hanks TD, Shadlen MN (2008) Bounded integration in parietal cortex underlies decisions even when viewing duration is dictated by the environment. J Neurosci 28:3017-3029.

Ludwig CJ, Gilchrist ID, McSorley E, Baddeley RJ (2005) The temporal impulse response underlying saccadic decisions. J Neurosci 25:9907-9912.

Milner AD, Goodale MA (2008) Two visual systems re-viewed. Neuropsychologia 46:774-785.

Whitney D, Cavanagh P (2000) Motion distorts visual space: shifting the perceived position of remote stationary objects. Nat Neurosci 3:954-959. 